\title{
Towards a unified brain theory
}

\author{
Lester Ingber \\ Physical Studies Institute, Inc., P.O. Box W; Solana Beach, CA 92075, USA \\ and Institute for Pure and Applied Physical Sciences, University of California San Diego, \\ La Jolla, CA 92093, USA
}

\begin{abstract}
An approach to collective aspects of the neocortical system is formulated by methods of modern non-equilibrium statistical mechanics. Microscopic neuronal synaptic interactions are first spatially averaged over columnar domains. These spatially ordered domains include well formulated fluctuations that retain contact with the original physical synaptic parameters. They also are a suitable substrate for macroscopic spatial-temporal regions described by Fokker-Planck and Lagrangian formalisms. This development clarifies similarities and differences among previous studies, suggests new analytically supported insights into neocortical function and permits future approximation or elaboration within current paradigms of collective systems.
\end{abstract}

\section{Introduction and rationale}

Relative to other biological entities, the intrinsic synaptic activity of the most highly evolved mammalian human neocortex functions via the most degenerate and the shortest-ranged neuronal interactions. This suggests that many collective aspects of this system may be fruitfully studied similarly to other collective physical systems. I.e. collective effects from clustering of neurons (Szentágothai \& Arbib, 1974) or from statistical interactions (Katchalsky, Rowland \& Blumenthal, 1974) are proposed to be mechanisms of information processing, in addition to the 'hard-wiring' mechanisms also possessed by other more ordered cortical entities (Afifi \& Bergman, 1980; Sommerhoff, 1974).

An approach has been recently formulated for this purpose (Ingber, in preparation). Three major divisions of this work, corresponding to Sections IIIA, B and C respectively, will be referred to as SMNI-I, -II, -III, respectively. The preliminary results of this formulation recommend further detailed study of neocortical interactions using this methodology. Insights are gained into neocortical function at several scales of interaction, applicable to other related disciplines as discussed in Sections II and IV. In this sense, the possibility of a 'unified brain theory' is suggested.

Reasonable criteria for any physical approach should include at least three basic Features in common with other collective physical systems.

\section{(A) Interactions}

Short-ranged neuronal interactions over time periods of several milliseconds should be derived from even more microscopic synaptic activites (Shepherd, 1977) [see Fig. 1(a)]. 


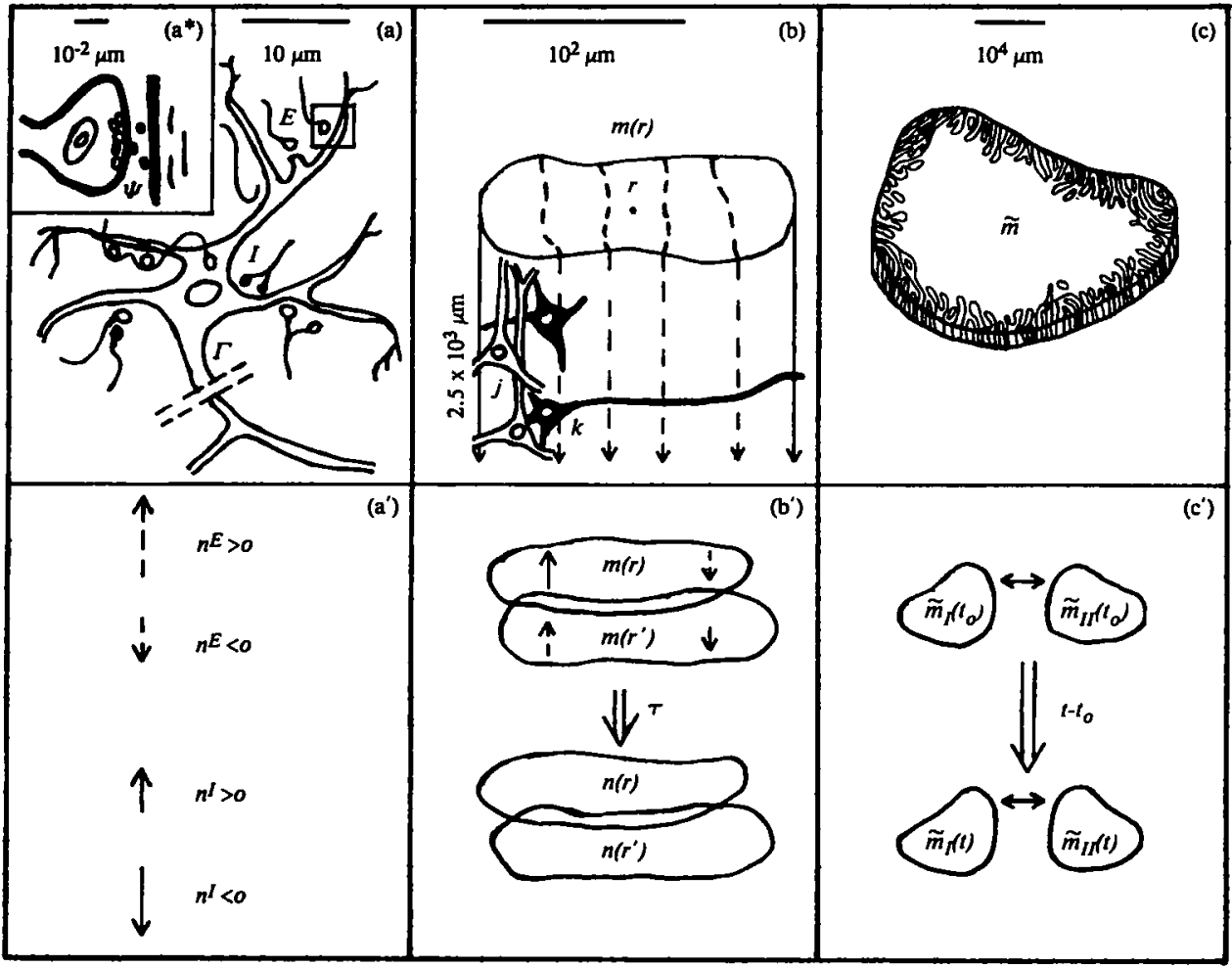

Fig. 1. Mlustrated are three biophysical scales of neocortical interactions: $(a),\left(a^{*}\right)$, $\left(a^{\prime}\right)$ - microscopic neurons; $(b),\left(b^{\prime}\right)-$ mesocolumnar domains; $(c),\left(c^{\prime}\right)$ - macroscopic regions. In $\left(a^{*}\right)$ synaptic inter-neuronal interactions, averaged over by mesocolumns, are phenomenologically described by the mean and the variance of a distribution $\Psi$. Similarly, in (a) intra-neuronal transmissions are phenomenologically described by the mean and variance of $\Gamma$. (This is a modified freehand sketch from Sommerhoff (1974).) Mesocolumnar-averaged excitatory (E) and inhibitory (I) neuronal firings, $n$ and $m$, are represented in $\left(a^{\prime}\right)$. In $(b)$ the vertical organization of mincolumns is sketched together with their horizontal stratification, yielding a physiological entity, the mesocolumn. In $\left(b^{\prime}\right)$ the overlap of interacting mesocolumns is sketched. In (c) macroscopic regions of neocortex are depicted as arising from many mesocolumnar domains. These are the regions designated for study here. $\left(c^{\prime}\right)$ sketches how regions may be coupled by long-ranged interactions

Long-ranged excitatory interactions from specific neuronal pathways, e.g. ipsilateral association, contralateral-commissural and thalamocortical fibers must be consistently treated. These long-ranged interactions are also very important for collective activity in mammalian cortex (Braitenberg, 1978) and it is possible to examine them within these paradigms. Longer-time, weaker and modulatory non-synaptic influences, e.g. humoral and electrotonic interactions (Dismukes, 1979; McGeer, Eccles \& McGeer, 1978; Schmitt, Dev \& Smith, 1976), are not presently explicitly included in this study.

\section{(B) Statistics}

Neurons separated by large distances, e.g. across $10^{3}$ to $10^{8}$ neurons, can be statistically coupled via the short-ranged interactions in (A) [see Fig. 1(c)]. Order parameters must be identified. Intrinsic fluctuations from the microscopic synaptic and neuronal systems, 
nonlinear diffusion effects, must be included (SMNI-II). The larger, but still relatively microscopic columnar system is referred to here as the 'mesoscopic' domain of interaction [see Fig. 1(b)]. Collective mechanisms of statistical firings of these regularly spatially ordered mesoscopic domains are closer to the observed anatomy and physiology of neocortex (Szentágothai \& Arbib, 1974), than to other theories hypothesizing random neural networks (Griffith, 1971; McCulloch \& Pitts, 1943).

Fluctuations represented by gradient couplings between these mesoscopic domains, familiar to other physical systems, may influence critical behavior in regions of second order phase transitions between ordered and disordered states (Stanley, 1971; Wilson \& Kogurt, 1974). Then the finite spatial 2-dimensionality, component number of order parameters, and symmetry aspects of the neocortical laminae must be explicitly formulated. Several kinds of order and excitations should be considered in this context (Barber, 1980). A non-technical treatment of critical phenomena may be found in Wilson (1979).

\section{(C) Dynamics}

A viable formulation must be adopted to describe the statistical time evolution of the mesoscopic non-equilibrium system over time scales of $10^{2}$ to $10^{4} \mathrm{~ms}$ (Haken, 1975, 1978; van Kampen, 1976). This feature is intimately connected with Feature (B) because the natural order parameters are dynamic firings of the mesoscopic system.

As SMNI-III has already established, it is reasonable to distinguish between neuronal mechanisms the neocortex uses to process information, e.g. electrical-chemical activity to be coded into higher order structures, from the structures and their relationships of information the neocortex processes (see Fig. 2). I.e. a Lagrangian, or 'potential', is derived which operates on firing states of the system. 'Information' is a well defined concept related to the probability eigenfunctions of this Lagrangian (Haken, 1978). The dynamic physical mechanisms of 'how' are correlated to the observation that minicolumns are a uniformly pervasive structure of neocortex (Mountcastle, 1978). This observation is analytically developed here by methods of modern statistical mechanics. Structural constraints on 'what' information is processed are thereby also obtained. Applications to specific evolutionary mechanisms often involves the coupling of the mechanisms and the structures, and so insights are also gained by appreciating this coupling.

As long as collective mechanisms arising in a physical system characterized by the above Features are considered to be viable sources of collective neocortical phenomena, then these Features must be correctly formulated, perhaps even before conjecturing about their biological evolutionary applications.

Although the level of co-operativity between distant neurons is quite low (Adey, 1978), macroscopic regions reflect co-operative behavior, conjectured here to best be understood as being initiated at the mesoscopic level of interaction. The empirical existence of collective spatial-temporal neocortical activity is supported by statistical analyses of electroencephalographic (John, 1972) and magnetoencephalographic (Williamson, Kaufman \& Brenner, 1979) recordings. Evoked potential studies (Hillyard, Hink, Schwent \& Picton, 1973; Kutas \& Hillyard, 1970; Näätanen, 1975) confirm the existence of macroscopic activity persisting for hundreds of msec (Dykes, 1978). Some studies, e.g. Nunez (1974), suggest that much of EEG data from electromagnetic potentials on the scalp can be explained essentially as dynamic dipole-layered excitatory interactions of the relatively low population of long-ranged excitatory interactions, only somewhat mediated by the short-ranged interactions. Although this hypothesis probably understates the degree to which EEG is a measure of neocortical activity, this does imply some EEG data may not be good 


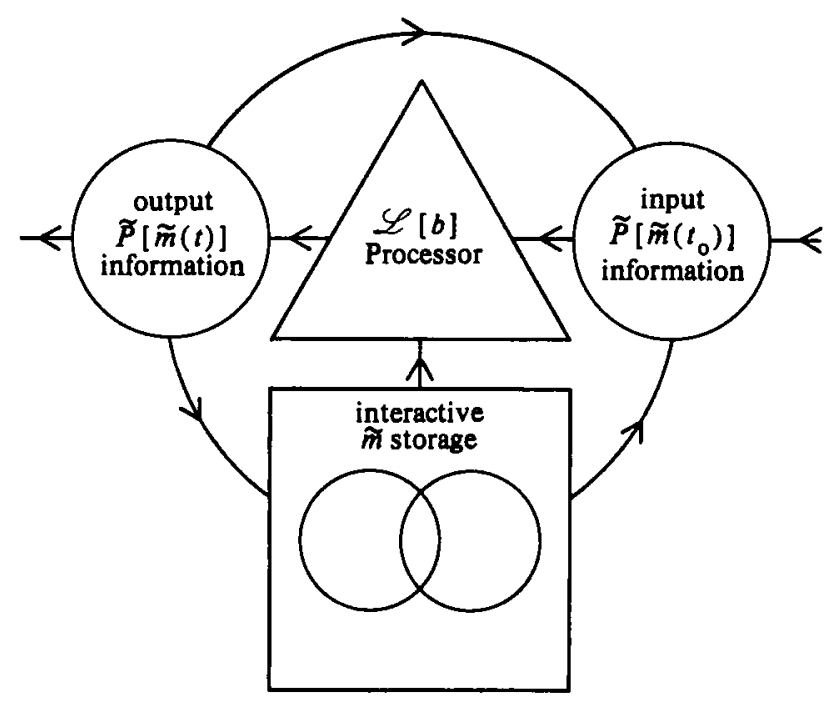

Fig. 2. Represented is the relationship of this study to other conjectured neurological and neuropsychological structures and processes. The macroscopic Lagrangian $\mathscr{L}$ in the triangle represents the interactions and fluctuations that process input to output patterns of mesocolumnar firings. These patterns, represented by the RHS and LHS circles are the probability eigenfunctions of $\mathscr{L}$. The $b$ 's are the expansion coefficients of the polynomial representation of $\mathscr{L}$. This formulation is conjectured to support description of storage of acquired and inherited patterns, the overlapping circles within the square. The square may interact back on the triangle, changing the electrical and chemical patterns which affect the local and global information processing of $\mathscr{L}$

physiological correlates of much of neocortical information processing which is certainly mediated by microscopic and mesoscopic interactions as treated in this study.

\section{Other studies}

\section{A. How information is processed}

There is a large literature dealing with neuronal mechanisms that intuits phenomenological differential Langevin-type equations from rates of change of average excitatory and inhibitory neuronal firings, and then proceeds to search for collective behavior, e.g. limit cycles, oscillatory behavior, etc. Comparisons with applications of similar Langevin, FokkerPlanck, and master equations to those used in other physical systems, e.g. as done by Haken (1975), illustrate that this pioneering application of these appropriate formalisms to the neocortical system still has much to offer. Much inspiration for these applications has come from the work in non-equilibrium thermodynamics of Prigogine (Nicolas \& Prigogine, 1973) and the intuition of Katchalsky (Katchalsky et al., 1974). The statistical mechanics formulation of similar systems is, however, somewhat less phenomenological and is found to be more useful for this study.

In applying this approach to neocortex, Wilson \& Cowan $(1972,1973)$ effectively intuit a set of deterministic Langevin equations, i.e. without including fluctuations, defined neuronal interactions, or synaptic statistics. Their approach neglects Features (A) and (B). The approach developed here can be further approximated and transformed to their equations, retaining some of these Features (Graham, 1977; Haken, 1975). Babloyantz \& Kaczmarek (Babloyantz \& Kaczmarek, 1979; Kaczmarek \& Babloyantz, 1977) have 
applied similar equations, e.g. to search for the onset of epileptic seizures. Freeman (1975, 1978) has intuited similar neuronal rate equations for the olfactory bulb. These analyses also lack inclusion of Features (A) and (B).

Takeuchi \& Amari (1979) have included other synaptic mechanisms in sets of differential rate equations, to study these effects on self-organization. Applications are also made to explain the ontogenesis of minicolumnar formations, similar in spirit, albeit with more detailed analytic development, to the pioneering work of von der Malsburg (1979) for ocularity domains (von der Malsburg \& Willshaw, 1977). These approaches also neglect Features (A) and (B). The approach developed here, besides including these Features, permits a more picturesque and full description of such phenomena in terms of peaks and valleys of potentials describing phase transitions.

Another approach, dating back to Cragg \& Temperley (1954) intuits an Ising-type model of neuronal interactions, and attempts to describe collective phases. This model was again intuited by Little (1974). This approach is incorrect for macroscopic, dynamic, nonequilibrium systems. Causality must be correctly formulated. E.g., such asymmetries between temporal and spatial dimensions are regularly noted even for macroscopic equilibrium systems (Ma, 1979). Shaw \& Vasudevan (1974) added Feature (A) to this approach, deriving the exponential expression of Little from the empirics of neuromuscular synaptic statistics. A simpler and more direct derivation is given in SMNI-II, which also generalizes the dependence of the final result on the type of statistics assumed for the quantal distribution of chemical transmitter between synapses. In subsequent papers, to facilitate their algebra, they drop all contributions to their neuronal transition rate that detract from a quadratic form (Little \& Shaw, 1978; Shaw \& Roney, 1979). However, these contributions are necessary to investigate first and second order phase transitions, (tri)critical points, etc. (Ma, 1976). Their continued analogy to a spatial Ising Hamiltonian, with an enormous number of nearest neighbors, is not practically useable in the formal studies they propose in Shaw \& Roney (1979). The key 'degeneracy factors' of Shaw \& Roney (1979) are straightforwardly derived as binomial coefficients in this study. These coefficients are shown to be dependent on the above-mentioned functional dependence of the transition rate on the postsynaptic firing rate, which is why care is to be taken in that derivation (SMNI-II). Although they neglect Features (B) and (C), this pioneering work has yielded several useful insights into the systematics of neuronal organization.

These above works also assume a more random connectivity among neurons that can be justified by observations of columnar anatomy and physiology (Hubel \& Wiesel, 1977; Mountcastle, 1978; Szentágothai \& Arbib, 1974). This study explicitly establishes an ordered 2-dimensional mesoscopic substrate upon which a macroscopic formulation of statistical firings is developed. Microscopic circuitries contributing to mesoscopic domains are not denied.

\section{B. What information is processed}

A large literature dealing with information processing of neural nets, applied to perception, learning and memory, typically intuits the existence of an algebra of information vectors isomorphic to a linear vector space (Amari, 1977; Anderson, 1972; Anderson, Silverstein, Ritz \& Jones, 1977; Birkhoff \& MacLane, 1953; Cooper, 1973). These studies also typically assume a more random connectivity than is empirically justified, as noted in Section IIA above.

The details of the structures of these vectors and of their relationships are based on the empirics of neuronal interaction, e.g. of hypothesized patterns of neuronal firings, but not nearly to the degree of analysis of the studies mentioned in Section IIA. Although more 
mechanisms and biological applications can be introduced and analysed by these studies than by those in Section IIA, the experimental and theoretical values of their conclusions are diminished because they do not reasonably draw empirical support and analytic development from relatively physical interactions, e.g. from Features (A), (B) or (C).

In this study, the sets of eigenfunctions of the physical system which includes Features (A), (B) and (C) may now reasonably be considered as candidates for the linear vector spaces previously assumed by these other studies. Intuitive insight, supported by an analytic formalism into the structure of these eigenfunctions is obtained from the potential calculated in this study. Future investigations will determine the degree of complementarity of this study with these other mechanisms and applications, thereby possibly enhancing their value as well as extending the utility of this theory.

These eigenfunctions arise quite naturally from a statistical mechanics analysis. No other esoteric concepts yet seem to be required to understand this process, contrary to other conjectures (Stuart, Takahashi \& Umezawa, 1978; Walker, 1970). Ironically, the history and structure of these quantum concepts (Jammer, 1974) argue well against using them for the neuronal system. Of course, a logical distinction must be drawn between the concepts peculiar to other (e.g. quantum) systems, and those mathematical techniques derived in those contexts, but which can be and are used to great benefit in formulating other systems such as the neocortex. The proposed mechanisms of Adey (1978) and Fröhlich (1977) may be exceptional circumstances invoking the physics of quantum mechanics, but even if they exist they are most likely not the main mechanisms of collective neocortical information processing.

\section{Present study}

\section{A. Microscopic neurons}

Fig. 1(a) illustrates the microscopic neuronal interaction scale on the order of several micrometers. Neocortical neurons typically have many dendrites that receive quanta of chemical postsynaptic stimulation from many other neurons. The distribution of quanta transmitted across synapses takes place on the scale of $10^{-2} \mu \mathrm{m}$, as illustrated in the inset Fig. 1( $\left.\mathrm{a}^{*}\right)$. Each quantum has thousands of molecules of chemical neurotransmitter than affect the chemically-gated postsynaptic membrane. Chemical transmissions in neocortex are believed to be either excitatory $(E)$, e.g. glutamic acid, or inhibitory $(I)$, e.g. $\gamma$-aminobutyric acid. There exist many transmitters as well as other chemicals that modulate their effects, but it is assumed that after millions of synapses on hundreds of neurons are averaged over, then it is reasonable to ascribe a distribution $\Psi$ with a mean and a variance for $E$ and $I$ interneuronal interactions.

This same averaging procedure makes it reasonable to ascribe a distribution $\Gamma$ with a mean and a variance for $E$ and $I$ intra-neuronal interactions. A Gaussian $\Gamma$ is taken to describe the distribution of electric polarizations caused by chemical quanta impinging on the postsynaptic membrane. These polarizations give a resultant polarization at the base of the neuron, the axon [extension in Fig. 1(a) cut by double broken line]. The base of the axon of a large neuron typically lies within a hillock and the axonal fiber may be myelinated. However, smaller neurons typically lack these distinguishing features. Experimental techniques are not yet sufficiently advanced to attempt the explicit averaging procedures necessary to establish the parameters of $\Psi$ and $\Gamma$ in vivo. Differential attenuations of polarizations from synapses to the base of an axon are here only phenomenologically accounted for by including these geometric and physiological effects into the mean and variance of $\Gamma$. 
With a sufficient depolarization of $\approx 10$ to $20 \mathrm{mV}$, within an absolute and relative refractory period of $\approx 5 \mathrm{~ms}$, an action potential is pulsed down the axon and its many collaterals, affecting voltage-gated presynaptic membranes to release quanta of neurotransmitters.

In the present study, it is assumed that the anatomical and chemical independence of excitatory depolarizations and inhibitory hyperpolarizations are well established in neocortex. This independence is retained throughout this study.

It should be noted that experimental studies used to infer $\Psi$ and $\Gamma$ at neuromuscular synaptic junctions were made possible by deliberately reducing the number of quanta by lowering external Ca concentrations (Boyd \& Martin, 1956; Katz, 1966). $\Psi$ was found to be Poisson, but in that system, where hundreds of quanta are transmitted in vivo, $\Psi$ may well be otherwise, e.g. Gaussian with an independent mean and variance. In neocortex, probably small numbers of quanta are transmitted at synapses, but other effects, e.g. non-linear summation of postsynaptic potentials etc. (Shepherd, 1979), may detract from simple Poisson statistics. This short discussion serves to point out possible differences in $\Psi$ from many sources. However, the derivation of the systematics of synaptic interactions given in SMNI-II makes it plausible that, for reasonable neuronal parameters, the statistical folding of $\Psi$ and $\Gamma$ is essentially independent of the functional form, not the numerical mean and variance, taken for $\Psi$.

\section{B. Mesoscopic columns}

At this stage, severe approximation and modelling would have to be, and is usually attempted to discuss further the macroscopic system. However, advantage can be taken of the empirically observed columnar structure (Goldman \& Nauta, 1977; Hubel \& Wiesel, 1962, 1977; Mountcastle, 1978; Szentágothai, 1974; Szentágothai \& Arbib, 1974) first analytically to scale the neuronal system into 'mesoscopic' domains that are still relatively microscopic to the mascroscopic regions to be described. This mesocolumnar structure effectively spatially averages the neuronal interactions within one to several firing periods. After some initial labor, columnar activity and fluctuations become easier to analytically and conceptually describe than the more complicated morass of synaptic interactions, thereby facilitating the macroscopic study.

The neocortex has $\approx 5 \times 10^{10}$ neurons distributed uniformly over $\approx 5 \times 10^{8}$ minicolumns. (The visual cortex has double this density.) Within these minicolumns, a 'vertical' structure is defined perpendicular to six laminae of total thickness $\approx 2.5 \times 10^{3} \mu \mathrm{m}$, principally by the efferent pyramidal cells. They exhibit vertical apical bundling of their dendrites in the upper laminae, and some of their recurrent axonal collaterals also ascend to upper laminae. A number of other fusiform, Matinotti and stellate cells, e.g. granule cells in sensory cortex and basket cells in motor cortex, also contribute to this vertical organization. In general laminae I to IV are receptive and laminae V and VI are efferent.

However, 'horizontal' dendritic basal arborizations of the pyramidal cells, tangential to the laminae, horizontal axonal collaterals of the pyramidal cells and horizontal processes of stellate, Martinotti and neonatal horizontal cells all impart horizontal stratification to columnar interactions. This stratification and other long-ranged common efferent input to groups of minicolumns, define a physiological unit that encompasses a number of minicolumns. Therefore, although the columnar concept has anatomical and physiological support, the minicolumnar boundaries are not so clearly defined (Dykes, 1978).

This study formalizes these circumstances by defining a 'mesocolumn' with extent $\approx 10^{2} \mu \mathrm{m}$, as an intermediate integral physiological unit encompassing one to several minicolumns [see Fig. 1(b)]. Dynamic nearest-neighbor interactions between mesocolumns are then analytically defined by their overlapping neuronal interactions [see Fig. 1( $\left.b^{\prime}\right)$ ]. This 
approach permits future analytic modification, as differences between inter- and intramesocolumnar interactions and circuitries become experimentally clarified.

The resulting picture of columnar interactions is relatively simpler than a mass of neurons, but not too simple to the point of uselessness. A collection of average excitatory and inhibitory neuronal firings now define a continuum of mesocolumnar firings, with a zeroeth order binomial distribution, also easily intuited by counting permutations of firings (SMNI-II). This analytically articulates the meaning of neuronal 'degeneracy'. The properly calculated distribution contains nearest-neighbor mesocolumnar interactions expressed as derivative correction terms. This decomposition is validated by numerical considerations of neocortical parameters in SMNI-II. Excitatory and inhibitory sensitivity to these interactions survives, similar to the sensitivity encountered by single neurons. In this manner, the microscopic effects of many types of neurons, synapses, neurotransmitters, cellular architecture, and circuitry, may be practically averaged for macroscopic considerations.

\section{Macroscopic regions}

Figs. 1(c) and $\left(c^{\prime}\right)$ illustrate how the mesocolumnar structure of Section IIB is a substrate for activity persisting for hundreds of milliseconds over a spatial region containing $\Lambda \approx 5 \times 10^{5}$ mesocolumns, i.e. $\Lambda \approx 10^{-2}$ of total cortical area of $4 \times 10^{11} \mu \mathrm{m}^{2}$. Extended regional intrinsic activity is possible, as conglomerates of 10 to 30 regions may interact (Mountcastle, 1978).

Given the mesocolumnar conditional transition probability, a master equation for the differential joint probability of all mesocolumns firing in the region can be developed from the transition probability calculated in SMNI-II. The existence of a continuous distribution for the range of mesocolumnar firings allows an expansion in the terms of the 'jump' ratemoments to be developed for further investigation (Kubo, Matsuo \& Kitahara, 1973; Nicolis \& Prigogine, 1973; van Kampen, 1976). This is still not necessarily a Markovian process, as can be seen by examining various temporal strings of conditional probabilities between an initial and final time, and summing over intermediate time periods. A short stochastic memory for the mesoscopic conditional probability does not necessarily imply the same for the macroscopic joint probability.

If the system is Markovian, a Fokker-Planck equation can be developed that expresses the differential evolution of the joint probability in terms of a macroscopic mean and variance; e.g. it is Gaussian. From this point, further transformation permits a set of Langevin equations to be derived describing rates of change of 'average' neurons (Graham, 1977a; Stratonovich, 1968; West, Bulsara, Lindenberg, Seshadri \& Shuler, 1979).

However, starting directly with the transition rates derived in SMNI-II a path-integral derivation of a Lagrangian is possible, which is essentially the integral of the differential propagator (Dekker, 1979, 1980; Grabert \& Green, 1979; Graham, 1977a, $b$; Feynman \& Hibbs, 1965; Langouche, Roekaerts \& Tirapegui, 1979; Onsager \& Machlup, 1953a,b) defining a Markovian, highly non-linear system. This permits the distinction between the mechanisms and the structures of information processing discussed previously. As such this is a very useful and analytic tool to investigate neocortex. Fokker-Planck and Langevin equations can be derived.

This Lagrangian can be expanded into or fit to a polynomial, and the resulting expression yields a generalized Ginzburg-Landau (G-L) expression for future studies (Haken, 1978; Wilson \& Kogurt, 1974). Although requiring substantial algebraic manipulation, this representation may make this work amenable to future time-dependent renormalization group studies of critical phenomena, which presently assume this G-L expression as a starting point, either in the Fokker-Planck or the Lagrangian representation (Brézin, Le Guillou \& Zinn-Justin, 1975; De Dominicus, 1975; De Dominicus, Brézin \& Zinn-Justin, 1975; Hohenberg \& Halperin, 1977; Ma, 1976; Mazenko, Nolan \& Valls, 1978; Niemeijer \& van Leeuwen, 1975). 
Examination of this G-L polynomial affords a conceptually picturesque result that is simpler and more concise than previous studies: Various possible shapes and interpretations of G-L polynomials, e.g. in phase transitions, limit cycles etc., are well described in Haken (1978). For example, if this potential would be reasonably described within a range of neuronal parameters by an increasing parabola, its minimum at zero firing would be the most probable state of the system. All firings are equally possible, giving this zero average. Its eigenfunctions would be the Hermite polynomials (Mathews \& Walker, 1970), with weights given by the mesocolumnar parameters. If a positive quartic term were added, and if the quadratic term became negative, then two non-zero stable states and a metastable state would exist, i.e. two valleys separated by a peak, and bifurcation, or collective behavior of all firings belonging to just one minima, could occur. A cubic term would introduce a first-order transition to the lowest state. Spatial and temporal gradient fluctuations determine just how well the system stays in these minima. In the absence of a cubic term, for a small linear term, critical behavior could occur when the neuronal parameters shift to a region such that the quadratic coefficient can go through zero. Fluctuations at all spatial and temporal scales may become relevant (Ma, 1976; Wilson, 1979). Interactions between ordered and disordered phases may account for interesting neocortical mechanisms.

Therefore, although the derivation of a G-L polynomial is not essential to calculate some macroscopic properties of the system as already developed by this study, this is presently essential for input into some other physical studies. This representation is also desirable for a first study, to derive intuitive insights.

For most collective systems, G-L expressions rest on intuitive models, in that a microscopic (e.g. quantum) treatment has not yet been analytically obtained. E.g. after much approximation, a G-L expression can be obtained for Ising-type systems (Wilson \& Kogurt, 1974). However, typically in these systems, G-L theory has predicted and described observable phenomena rather well, and it accords so well with similar treatments of other collective systems that can be derived analytically from microscopic treatments, that G-L theory is aptly there considered a good physical approach. Neocortical studies have not achieved such a wealth of data, and so the burden is presently more on theory to establish its existence. Conversely, similar analytic development of a G-L expression from relatively microscopic principles is a luxury afforded to only a few physical systems.

\section{Preliminary results and conjectures}

\section{A. Calculations}

To calculate $\mathscr{L}$ in a G-L polynomial form, a computer code to keep track of all expansion coefficients has been written to calculate the expansion coefficients. SURFACE II (Sampson, 1978 ) is used to portray the surface of the potential in $m^{G}-\mathscr{L}$ space ( $G$ represents $E$ or $I$ firings). These results are still quite tentative and more computations must yet be done.

However, some preliminary results have been obtained. Typically, contributions to the Lagrangian come from the drift and diffusion, and the differential propagator includes some other entities peculiar to Riemannian geometry (Weinberg, 1972), e.g. the curvature and covariant derivatives of the drift and diffusion in $m^{G}$-space. This mathematics is used to derive the non-linear Lagrangian from the short-time differential propagator. These entities are typically important in the sensitive region of zero contribution to the drift from just previous firings, the region of maximum degeneracy of firing states (SMNI-II).

For sets of mesoscopic parameters derived from reasonable ranges of neuronal parameters, points on the surface of the Lagrangian are calculated. Microscopic, mesoscopic and macroscopic (e.g. Riemannian geometric features) effects of the neocortical system put 'dents', i.e. 
local minima, in this surface. Depending on the strengths of fluctuations and driving forces, phase transitions between these minima are possible, providing varying degrees of ordered firings or non-firings of $m^{E}$ and $m^{I}$. Even for a simple choice of mesocolumnar parameters that yield a zero mean drift at a local minimum of zero $m^{G}$, the system is still not simply disordered, due to the possibility of phase transitions to other nearby minima. Other sets of parameters typically do not even support a local minimum at zero $m^{G}$. More technical details are given in SMNI-III.

\section{B. Applications}

The following conjectured applications demonstrate how a proposed 'unified brain theory' may yet evolve to explain varied phenomena at many different spatial-temporal scales, spanning many disciplines.

1. Hysteresis effects in information processing, treated as a first order phase transition with fluctuations can test the preliminary results of Wilson (1977).

2. Reaction and adaptation of the system to input patters can test the intuitions of models of information processing (Szentágothai \& Arbib, 1974; Takeuchi \& Amari, 1979).

3. The small scale of the neocortical system about which the system fluctuates is derived to be $N^{-1}$, the inverse of the number of neurons in a mesocolumn. This can be crudely interpreted as the effective 'temperature' or inherent noise of the system. The sharpness of the step-function contribution to the mean mesocolumnar firing is sensitive to a factor of $N^{1 / 2}$. Additionally, the strength of coupling between mesocolumns scales as $N^{3 / 2}$. Thus the size of mesocolumns directly affects the breadth and depth of the information processing capability of neocortex. It is interesting to note that human visual cortex, which might be assumed to require the finest tuning in neocortex, is unique in having twice the number of neurons per minicolumn than other areas of neocortex (Mountcastle, 1978). An analytic and calculable conjecture of one effect of aging is that although columnar integrity remains, the 'filtering' capability of the Lagrangian decreases as the total number of neurons also decreases.

4. Regions where the probability eigenfunctions become localized reflect potential large-scale collective activity. This may be one mechanism of epilepsy. The importance of fluctuations can be added to Kaczmarek \& Babloyantz (1977).

5. Ontogenesis of columnar structure may be described. In regions of phase transition, spatial-temporal pattern formation (Katchalsky et al., 1974) may provide some clues as to the nature of columnar development (Takeuchi \& Amari, 1979; von der Malsburg, 1979). It is interesting to speculate on whether the ontogenesis of the size $N$ is primarily fixed by local constraints of neuronal interactions, by finite global constraints of competitions between having large $N$ as well as large numbers of mesocolumns/region, or by constraints imposed by true homogeneous macroscopic spatial-temporal collective states of firings. This latter possibility further invites speculation that the morphogenesis of columnar structure is induced by the evolutionary desirability of permitting phase transitions between local and global information processing. This would also put constraints on the developing neonatal microscopic neuronal parameters.

6. The introduction of a dependence on repeated firings may allow an investigation into Hebb-type mechanisms for learning (Hebb, 1949; Takeuchi \& Amari, 1979). Takeuchi \& Amari (1979) also suggest modifications of synaptic strengths that have other applications. This formalism adds the features of fluctuations, and its parameters are relatively directly expressed by observable synaptic activities. Specific models of perception, learning and memory can be calculated.

7. The existence of local and global information processing in disordered and ordered 
phases, respectively, may support the observations of two independent attentional mechanisms (Schneider \& Shiffrin, 1977; Shiffrin \& Schneider, 1977). A study of critical regions may yield insights into their interactions.

8. A long-range goal is to establish a sound scientific basis for improving educational methods and institutions. Two years ago, I completed an eight-year project, administrating and teaching in an alternative junior-senior high school sponsored by the Institute for the Study of Attention, a subsidiary of the Physical Studies Institute (Ingber, 1972, 1981). We used the working hypothesis that local and global attention archetypes are inherent in all complex information processing, to develop an educational system to train teachers and students in over 30 academic, fine arts and physical disciplines (Ingber, 1976). These experiences support the conjecture that optimal use of these attention processes enhance learning and creativity. Circumstances most favorable to optimal use may occur at a critical point(s).

9. A quite tentative hypothesis is that there exists a specific innately developed pattern, e.g. a 'schema of self-awareness', that primarily monitors/screens specific (vital) functions, but that also has evolutionarily developed the capability to interact as a pattern with other information being processed by the above attentional processes, thereby possibly directing or even interfering with them. These physical mechanisms may circumvent criticisms usually levelled at such theories of consciousness and self-awareness (Rigterink, 1979).

\section{Acknowledgements}

I thank Michael Arbib for his critique of this work, and for his suggestion to write this paper.

\section{References}

Adey, W. R. (1978). Long-range electromagnetic field interactions at brain cell surfaces. In Magnetic Field Effect on Biological Systems. New York: Plenum. pp. 56-80.

Afifi, A. K. \& Bergman, R. A. (1980). Basic Neuroscience. Baltimore, MD: Urban \& Schwarzenberg. $519 \mathrm{pp}$.

Amari, S. -I. (1977). Neural theory of association and concept formation. Biol. Cyber. 26, $175-185$.

Anderson, J. A. (1972). A simple neural network generating an interactive memory. Math. Biosci. 14, 197-220.

Anderson, J. A., Silverstein, J. W., Ritz, S. A. \& Jones, R. S. (1977). Distinctive features, categorical perception and probability learning: Some applications of a neural model. Psych. Rev. 84, 413-451.

Babloyantz, A. \& Kaczmarek, L. K. (1979). Self-organization in biological systems with multiple cellular contacts. Bull. math. Biol. 41, 193-201.

Barber, M. N. (1980). Phase transitions in two dimensions. Phys. Rep. 59C, 375-409.

Birkhoff, G. \& MacLane, S. (1953). A Survey of Modern Algebra. Revised ed. New York: MacMillan. $472 \mathrm{pp}$.

Boyd, I. A. \& Martin, A. R. (1956). The end-plate potential in mammalian muscle. J. Physiol. (London) 132, 74-91.

Braitenberg, V. (1978). Cortical architectonics: general and areal. In (M. A. B. Brazier \& H. Petsche, Eds) Architectonics of the Cerebral Cortex. New York: Raven Press, pp. 443465.

Brézin, E., Le Guillou, J. C. \& Zinn-Justin, J. (1975). Field theoretical approach to critical phenomena. In (C. Domb \& M. S. Green, Eds) Phase Transitions and Critical Phenomena, Vol. 6. New York: Academic Press. pp. 125-247.

Cooper, L. N. (1973). A possible organization of animal memory and learning. In (B. Lundqvist \& S. Lundqvist, Eds) Collective Properties of Physical Systems. New York: Academic Press. pp. 252-264. 
Cragg, B. G. \& Temperley, H. N. V. (1954). The organization of neurones: A co-operative analogy. Electroenceph. clin. Neurophysiol. 6, 85-92.

De Dominicus, C. (1975). A Lagrangian version of Halperin-Hohenberg-Ma models for the dynamics of critical phenomena. Nuovo Cimento Lett. 12, 567-574.

De Dominicus, C., Brézin, E. \& Zinn-Justin, J. (1975). Field-theoretical techniques and critical dynamics. I. Ginzburg-Landau stochastic models without energy conservation. Phys. Rev. B 12, 4945-4953.

Dekker, H. (1980). Path integrals in Riemannian spaces. Phys. Lett. 76 A, 8-10.

Dismukes, R. K. (1979). New concepts of molecular communication among neurons. Behavl. brain Sci. 2, 409-448.

Dykes, R. W. (1978). The anatomy and physiology of the somatic sensory cortical regions. Proq. Neurobiol. 10, 33-88.

Feynman, R. P. and Hibbs, A. R. (1965). Quantum Mechanics and Path Integrals. New York: McGraw-Hill. 365 pp.

Freeman, W. J. (1975). Mass Action in the Nervous System. New York: Academic Press. $496 \mathrm{pp}$.

Freeman, W. J. (1978). Spatial properties of an EEG event in the olfactory bulb and cortex. Electroenceph. clin. neurophysiol. 44, 586-605.

Fröhlich, H. (1977). Possibilities of long and short range order interactions of biological systems. Neurosci. Res. Proq. Bull. 15, 67-72.

Goldman, P. S. \& Nauta, W. J. H. (1977). Columnar distribution of cortico-cortical fibers in the frontal association, limbic, and motor cortex of the developing rhesus monkey. Brain Res. 122, 393-413.

Grabert, H. \& Green, M. S. (1979). Fluctuations and nonlinear irreversible processes. Phys. Rev. $A$ 19, 1747-1756.

Graham, R. (1977a). Covariant formulation of non-equilibrium statistical thermodynamics. Z. Phys. B 26, 397-405.

Graham, R. (1977b). Lagrangian for diffusion of curved phase space. Phys. Rev. Lett. 38, 51-53.

Griffith, J. S. (1971). Mathematical Neurobiology. New York: Academic Press. $161 \mathrm{pp}$.

Haken, H. (1975). Cooperative phenomena in systems far from thermal equilibrium and in nonphysical systems. Rev. mod. Phys. 47, 67-121.

Haken, H. (1978). Synergetics. 2nd ed. New York: Springer-Verlag. 355 pp.

Hebb, D. O. (1949). The Organisation of Behavior. New York: Wiley. 335 pp.

Hillyard, S. A., Hink, R. F., Schwent, V. L. \& Picton, T. W. (1973). Electrical sign of selective attention in the human brain. Science 182, 177-180.

Hohenberg, P. C. \& Halperin, B. I. (1977). Theory of dynamic critical phenomena. Rev. mod. Phys. 49, 435-479.

Hubel, D. H. \& Wiesel, T. N. (1962). Receptive fields, binocular interaction and functional architecture in the cat's visual cortex. J. Physiol. 160, 106-154.

Hubel, D. H. \& Wiesel, T. N. (1977). Functional architecture of macaque monkey visual cortex. Proc. R. Soc. B 198, 1-59.

Ingber, L. (1972). Editorial: Learning to learn. Explore 7, 5-8.

Ingber, L. (1976). The Karate Instructor's Handbook. Solana Beach, Ca: ISA. 96 pp.

Ingber, L. (1981). Attention, physics and teaching. J. social biol. Struct. 4, 225-235.

Jammer, M. (1974). The Philosophy of Quantum Mechanics. New York: Wiley. 536 pp.

John, E. R. (1972). Switchboard versus statistical theories of learning and memory. Science $177,850-864$.

Kaczmarek, L. K. \& Babloyantz, A. (1977). Spatiotemporal patterns in epileptic seizures. Biol. Cyber. 26, 199-208.

Katchalsky, A. K., Rowland, V. \& Blumenthal, R. (1974). Dynamic patterns of brain assemblies. Neurosci. Res. Proq. Bull. 12, 1-187.

Katz, B. (1966). Nerve, Muscle, and Synapse. New York: McGraw-Hill. 193 pp.

Kubo, R., Matsuo, K. \& Kitahara, K. (1973). Fluctuation and relaxation of macrovariables. J. stat. Phys. 9, 51-96.

Kutas, M. \& Hillyard, S. A. (1980). Reading senseless sentences: Brain potentials reflect semantic incongruity. Science 207, 203-205.

Langouche, F., Roekaerts, D. \& Tirapegui, E. (1979). Discretization problems of functional integrals in phase space. Phys. Rev. D 20, 419-432. 
Little, W. A. (1974). The existence of persistent states in the brain. Math. Biosci. 19, 101-120.

Little, W. A. \& Shaw, G. L. (1978). Analytic study of the memory storage capacity of a neural network. Math. Biosci. 39, 281-290.

Ma, S. -K. (1976). Modern Theory of Critical Phenomena. Reading, MA: Benjamin/Cummings. $561 \mathrm{pp}$.

Ma, S. -K. (1979). Alternative approach to the dynamic renormalization group. Phys. Rev. $B$ $19,4824-4837$.

Mathews, J. \& Walker, R. L. (1970). Mathematical Methods of Physics. 2nd ed. New York: Benjamin. $501 \mathrm{pp}$.

Mazenko, G. F., Nolan, M. J. \& Valls, O. T. (1978). Application of the real-space renormalization group to dynamic critical phenomena. Phys. Rev. Lett. 41, 500-503.

McCulloch, W. S. \& Pitts, W. (1943). A logical calculus of the ideas immanent in nervous activity. Bull. Math. Biophys. 12, 207-219.

McGeer, P. C., Eccles, J. C. \& McGeer, E. G. (1978). Molecular Neurobiology of the Mammalian Brain. New York: Plenum. 644 pp.

Mountcastle, V. B. (1978). An organizing principle for cerebral function: The unit module and the distributed system. In (G. M. Edelman \& V. B. Mountcastle, Eds) The Mindful Brain. Cambridge: MIT Press, pp. 7-50.

Näätanen, R. (1975). Selective attention and evoked potentials in humans - a critical review. Biol. Psych. 2, 237-307.

Nicolis, G. \& Prigogine, I. (1973). Self-Organization in Nonequilibrium Systems. New York: Wiley. $491 \mathrm{pp}$.

Niemeijer, Th. \& van Leeuwen, J. M. J. (1975). Renormalization theory for Ising-like spin systems. In (C. Domb \& M. S. Green, Eds) Phase Transitions and Critical Phenomena. Vol. 6. New York: Academic Press. pp. 425-505.

Nunez, P. L. (1974). The brain equation: A model for the EEG. Math. Biosci. 21, 279-297.

Onsager, L. \& Machlup, S. (1953a). Fluctuations and irreversible processes. Phys. Rev. 91, $1505-1512$.

Onsager, L. \& Machlup, S. (1953b). Fluctuations and irreversible processes. II. Systems with kinetic energy. Phys. Rev. 91, 1512-1515.

Rigterink, R. (1979). Sperry's modified concept of consciousness. Cognition brain Theor. 3 , 63-67.

Sampson, R. J. (1978). SURFACE II Graphics System, revision one. Lawrence, KA: Kansas Geological Survey. 240 pp.

Schmitt, F. O., Dev, P. \& Smith, B. H. (1976). Electrotonic processing of information by brain cells. Science 193, 114-120.

Schneider, W. \& Shiffrin, R. M. (1977). Controlled and automatic human information processing: I. Detection, search, and attention. Psych. Rev. 84, 1-66.

Shaw, G. L. \& Roney, K. J. (1979). Analytic solution of a neural network theory based on an Ising spin system analogy. Phys. Lett. 74A, 146-150.

Shaw, G. L. \& Vasudevan, R. (1974). Persistent states of neural networks and the random nature of synaptic transmission. Math. Biosci. 21, 207-218.

Shepherd, G. M. (1979). The Synaptic Organization of the Brain. 2nd ed. New York: Oxford University Press. $436 \mathrm{pp}$.

Shiffrin, R. M. \& Schneider, W. (1977). Controlled and automatic human information processing. II. Perceptual learning, automatic attending, and a general theory. Psych. Rev. 84, 127-190.

Sommerhoff, G. (1974). Logic of the Living Brain. New York: Wiley. 413 pp.

Stanley, H. E. (1971). Introduction to Phase Transitions and Critical Phenomena. New York: Oxford University Press. 308 pp.

Stratonovich, R. L. (1968). Conditional Markov Processes and Their Application to the Theory of Optimal Control. New York: Elsevier, $347 \mathrm{pp}$.

Stuart, C. I. J. M., Takahashi, Y. \& Umezawa, H. (1978). On the stability and non-local properties of memory. J. theor. Biol. 71, 605-618.

Szentágothai, J. (1975). The "module-concept" in cerebral cortex architecture. Brain Res. 95, 475-496.

Szentágothai, J. \& Arbib, M. A. (1974). Conceptual models of neural organization. Neurosci. Res. Prog. Bull. 12,307-510.

Takeuchi, A. \& Amari, S. (1979). Formation of topographic maps and columnar micro- 
structures in nerve fields. Biol. Cyber. 35, 63-72.

van Kampen, N. G. (1976). Fluctuations in closed and open non-linear systems. In (L. Pál and P. Szépfalusy, Eds) Statistical Physics. Amsterdam: North-Holland. pp. 29-48.

van der Malsburg, Ch. (1979). Development of ocularity domains and growth behavior of axon terminals. Biol. Cyber. 32, 49-62.

van der Malsburg, Ch. \& Willshaw, D. J. (1977). How to label nerve cells so that they can interconnect in an ordered fashion. Proc. natn. Acad. Sci. USA 74, 5176-5178.

Walker, E. H. (1970). The nature of consciousness. Math. Biosci. 7, 131-178.

Weinberg, S. (1972). Gravitation and Cosmology. New York: Wiley. 657 pp.

West, B. J., Bulsara, A. R., Lindenberg, K., Seshadri, V. \& Shuler, K. E. (1979). Stochastic processes with non-additive fluctuations. I. Itô and Stratonovich calculus and the effects of correlations. Physica 97A, 211-233.

Williamson, S. J., Kaufman, L. \& Brenner, D. (1979). Evoked neuromagnetic fields of the human brain. J. appl. Phys. 50, 2418-2421.

Wilson, H. R. (1977). Hysteresis in binocular grating perception: Contrast effects. Vision Res. 17, 843-851.

Wilson, H. R. \& Cowan, J. D. (1972). Excitatory and inhibitory interactions in localized populations of model neurons. Biophys. J. 12, 1-23.

Wilson, H. R. \& Cowan, J. D. (1973). A mathematical theory of the functional dynamics of cortical and thalamic nervous tissue. Kybernetik 13, 55-80.

Wilson, K. G. (1979). Problems in physics with many scales of length. Scient. Am. 241, 158-179.

Wilson, K. G. \& Kogurt, J. (1974). The renormalization group and the $\epsilon$ expansion. Phys. Rep. C 12, 75-200. 